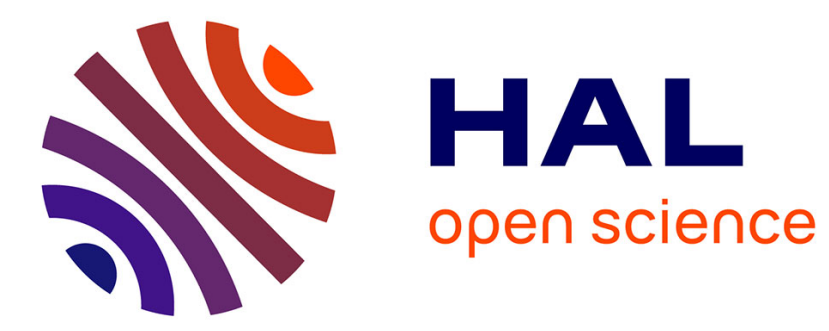

\title{
Development framework for a patient-centered record
}

John Puentes, Michèle Roux, Julien Montagner, Laurent Lecornu

\section{To cite this version:}

John Puentes, Michèle Roux, Julien Montagner, Laurent Lecornu. Development framework for a patient-centered record. Computer Methods and Programs in Biomedicine, 2012, 108, pp.1036 - 1051. 10.1016/j.cmpb.2012.06.007 . hal-01162673

\section{HAL Id: hal-01162673 https://hal.science/hal-01162673}

Submitted on 8 Jun 2021

HAL is a multi-disciplinary open access archive for the deposit and dissemination of scientific research documents, whether they are published or not. The documents may come from teaching and research institutions in France or abroad, or from public or private research centers.
L'archive ouverte pluridisciplinaire HAL, est destinée au dépôt et à la diffusion de documents scientifiques de niveau recherche, publiés ou non, émanant des établissements d'enseignement et de recherche français ou étrangers, des laboratoires publics ou privés. 


\title{
Development framework for a patient-centered record
}

\author{
John Puentes ${ }^{a, *}$, Michèle Roux ${ }^{b}$, Julien Montagner ${ }^{a}$, Laurent Lecornu ${ }^{a}$ \\ a Institut Mines-Telecom, Telecom Bretagne, Département Image et Traitement de l'Information, Brest, France \\ ${ }^{\mathrm{b}}$ Laboratoire Information Coordination Incitations, Université de Bretagne Occidentale, Brest, France
}

\author{
Keywords: \\ Patient-centered record \\ User requirements \\ Medical workflow \\ Complementary patient \\ information \\ Development methodology \\ Conceptual data model
}

\begin{abstract}
Patient records have been developed to support the physician-oriented medical activity scheme. One recommended yet rarely studied alternative, expected to improve healthcare, is the patient-centered record. We propose a development framework for such record, which includes domain-specific database models at the conceptual level, analyzing the fundamental role of complementary information destined to ensure proper patient understanding of related clinical situations. A patient-centered awareness field study of user requirements and medical workflow was carried out in three medical services and two technical units to identify the most relevant elements of the framework, and compared to the definitions of a theoretical approach. Three core data models - centered on the patient, medical personnel, and complementary patient information, corresponding to the determined set of entities, information exchanges and actors roles, constitute the technical recommendations of the development framework. An open source proof of concept prototype was developed to show the model feasibility. The resulting patient-centered record development framework implies particular medical personnel contributions to supply complementary information.
\end{abstract}

\section{Introduction}

Physicians practice relies on a particular combination of essential skills: clinical assessment based on interviewing, history taking, and examination, in addition to highly developed visual and explicit memory, combined with the use of a very large specialized terminology. These competences are supported by a strong professional culture based on paper patient records, often handled in a clinical workflow on which the patient moves from one step to the next of the healthcare process, as a passive subject under the responsibility of a physician or a care team. An alternative to this physician oriented scheme is the so-called patient-centered care, on which significant importance is given to the patient's understanding of the pathology, its diagnosis, treatment, and the way to cope with it, by means of complementary information that should be associated to the record. Notwithstanding the complexity of defining the patient-centered character of a medical interaction, essential elements to conceptualize it have been theoretically studied by Mead and Bower [1]. This approach relies on five dimensions: the explanation of an illness taking into account biomedical, psychologi$\mathrm{cal}$, and social factors; understanding the personal disease experience of each patient; an egalitarian physician-patient relationship including information availability and common decision making; a shared comprehension of the therapeutic objectives; and physician awareness of the potential impact of personal qualities and subjectivity on the relation with the patient. Most of these dimensions require selected complementary medical and legal information, as well as related lifestyle advice, not easily available in the information systems used by the conventional physician-centered approach.

* Corresponding author at: Telecom Bretagne, Image and Information Processing, CS 83818, 29238 Brest Cedex 3, Finistere, France. Tel.: +33229001339.

E-mail address: John.Puentes@telecom-bretagne.eu (J. Puentes). 
Despite the fact that patient-centeredness is eminently a medical practice notion, adapted applications based on information technology are still required in order to facilitate its transfer to medical activity support systems [2]. Even so, the current uses of information technology to functionally improve the physician-centered scheme evolve slowly, are somewhat limited, and face multiple challenges [3]. One of the core issues is related to electronic patient record structure and content, which has been the subject of multiple proposals for more than 35 years [4-9]. Nevertheless, no consensus has been reached between user requirements and system designers' initiatives. Furthermore, the patient record structure problem has been frequently unsuitably formulated in terms of new available proprietary technologies, instead of concentrating the effort on user requirements understanding and system compatibility with medical practice.

Currently, the electronic health record (EHR) is generated assembling data from different proprietary components of the hospital information systems (HIS), creating a considerable EHR architecture variability between healthcare institutions, as well as multiple interoperability issues. EHR models have been studied in detail according to functionality, structure, services accessibility, media support, and security [10]; defining how EHR architectures are significantly shaped by the medical specialty [11,12]; and with a particular focus on architectures using standardized communications of health data by combining reference models and archetypes [13], like openEHR [14] and ISO EN 13606 [15]. Even if the global openEHR archetypes architecture has been integrated by ISO EN 13606 and HL7 common clinical documents architecture [16], with the objective of seamless interoperability, the archetype software entities remain complex to develop [11]. Other models, like the American Society for Testing and Materials Standard ASTM E $1384[17,18]$, include in the EHR basic health information like patient identification, clinical history, laboratory tests results, diagnosis reports, drugs and treatments prescriptions, among others, in the form of data elements or documents transferable to compatible systems. None of these EHR models has been conceived to structure the required complementary information of the patient-centered approach, or to support direct patient access to it. On the other hand, initiatives as the personal health record (PHR) mainly concern systems that handle manually or automatically entered data, collected and managed by the patient, having eventual access to selected content of the EHR, but without specification of the tools necessary to understand these personal data and act on the information contained in the record [19]. Otherwise, the patient-centered record has been defined as a patient-owned and patient managed personal record, representing the health information important to patients in ways they prefer to represent it [20], which is somewhat similar to the PHR. Also, the patient-centered record should contain, besides the EHR information, a description of the assessment and plan, as well as attending notes on teaching services, in order to address the patient's perspective, beyond the disease [21]. Nonetheless, a specific computer model or development framework has not been proposed to implement such patient-centered record approaches.

In this work, a patient-centered record is a system designed to provide complementary information, directly associated to the medical data required by clinical practice. Such complementary information is dynamically adapted to the patient evolving situation, to support the patient's understanding and participation in the medical decision process. In that sense, it can encompass, but differ from the so-called consumer health information, conceived for patients already taking care of their own condition, with a particular interest in prevention and wellness. Collected complementary information is intended to enable patients to completely and easily understand their medical conditions and healthcare process, and be used mostly by the patient and family members. Therefore, it is not a PHR because there is neither personally collected data nor patient management of the complementary information, even though the proposed patient-centered record can be interconnected to the EHR. In addition, HIS have been traditionally designed for the most part to support administrative tasks and, in a slighter degree, healthcare professionals' activity [22]. Beyond this well known functional profile, HIS advancement is expected to also include a patient-centered record [23], adding undoubtedly to the complexity of how to develop adapted patient records. Our work focuses therefore on the question of how to conceive a realistic and extensible patient-centered record, adapted to patient requirements and the corresponding medical practice, capable of providing dynamically the necessary complementary patient information (CPI).

Although Mead and Bower approach seems adapted to evaluate physicians' self-perceived degree of patientcenteredness [24], it was not defined to take account of patient-centered record's role, and neither did other works that preceded it [25-27]. Yet, it provides a substantial reference to determine on which of its dimensions a patient-centered record is likely to be essential (Section 2). We compared those elements to the main related information exchanges identified by means of a patient-centered awareness field work, and the corresponding user requirements, to understand this workflow compatibility with a patient-centered practice (Section 3). Considering that CPI intends to provide detailed explanations of pathology diagnosis, main and alternative treatments, recommended changes of life style, as well as personal and legal advice, data of the patient-centered record were then modeled to cope with identified user requirements. Domain-specific database schemas defined at the conceptual level were used, matching the related patient-centered workflow and three particular entities: patient, medical personnel, and CPI. These schemas are presented as essential design and development recommendations, in the context of a patient-centered record proof of concept system (Section 4). The discussion focuses on the resulting development framework for a patient-centered record, as well as the database schemas conceptualization issues (Section 5), before outlining the learned lessons (Section 6) and future plans (Section 7).

\section{Background}

While patient-centered care appears as an intelligible medical practice oriented notion, it is less so for a patient-centered electronic record. From a medical informatics point of view, 
"patient-centeredness" remains a concept that needs real meaning [28]. However, such definition is not likely to rely on established clinical documentation practices, which do not provide optimal and integrated information necessary to build a patient-centered record [29]. On the other hand, efforts to associate patient-centered care and related technology have concentrated on making existing patient information accessible, for instance facilitating the use of a PHR [30-32], or identifying patient information access preferences [33]. Nevertheless, an explicit patient-centered record development framework has not been formulated.

Considering that the main interest of the patient-centered record is to enhance patient understanding and participation in the healthcare process, we first examined Mead and Bower theoretical analysis, before confronting it to a patientcentered awareness field study, in order to formulate the basic elements of the proposed development framework.

\subsection{Theoretical patient-centeredness}

Among the five dimensions of Mead and Bower approach [1], three rely strongly on information exchange, evidencing the important role that a patient-centered record could achieve:

- The explanation of an illness according to biomedical, psychological, and social factors.

- An egalitarian physician-patient relationship based on information availability and common decision making.

- A shared comprehension of the therapeutic objectives.

Explaining an illness from different professional perspectives - biomedical, but also psychological, and social-requires an exchange of selected information, in agreement with patients' questions and beyond essential points discussed with the physician during consultation. Such ancillary information should be provided to the patient and/or relatives, to ensure them a fine understanding of the patient's medical situation. This element seems essential to enable common decision making, even though it is not straightforward to determine how much information would compensate the evident knowledge unbalance between the physician and the patient. That is also the case of fundamental information to facilitate proper comprehension of defined therapeutic objectives. Regardless of this implicit content variability, our work searched to define a coherent development framework of a patient-centered record giving access to such kind of information, taking into account the previously identified three dimensions of the Mead and Bower model as starting premises, to be confronted with a field study carried out at medical services and units.

\subsection{Prospective patient-centeredness according to information exchange}

To study system requirements in detail, three hospital medical services (general medicine, gastroenterology, and pneumology) and two technical units (orthopedic surgery and nuclear medicine), were visited during 2 days by two analysts, to examine prospective patient-centered record users' needs, following daily service personnel (specialist, nurse,

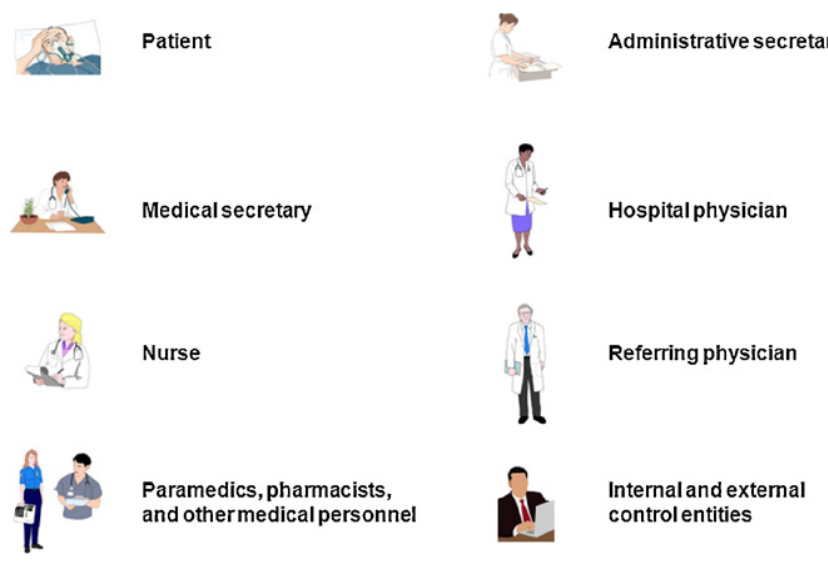

Fig. 1 - Schematic representation of the main patient-centered workflow actors.

secretaries) activities, and defining the resulting workflows in a hypothetical patient-centered scenario. On the other hand, 12 volunteer patients (at least 2 per service or unit) were asked to state what their patient-centered record could contain. Even though to answer such open question all patients referred to understandable and accessible information about diagnosis, treatment, expected results and medication secondary effects, only 5 added details like advice on alternative treatments, life style and legal advice.

Considering the lack of systemic analysis to develop patient-centered records, users' needs were analyzed with respect to observed daily activities, investigating which changes would be necessary to establish a patient-centered record information flow. The interaction context inside and between medical services and technical units was identified as the reference workflow scenario, given that those interactions encompass most of the system requirements, detailed in the next section. This choice restricts both involved personnel and information streams, permitting to examine possible variations of the conventional medical service and technical unit workflow, when a patient-centered perspective is applied. Besides the patient, seven hospital staff members were identified as the main patient-centered workflow actors (Fig. 1), with high potential of having an active role related to the patientcentered record.

These actors' activities are strongly interrelated, mostly based on information exchanges (Fig. 2), complying with the three underlined dimensions of Mead and Bower model. A visit to the family physician starts the exchanges, leading after consultation to refer the patient to the hospital physician, indicating in a letter the object of the visit, and requesting an appointment to the medical secretary. Meanwhile the patient may have been aided by paramedics, who transmit elements about their intervention, before contacting the medical and administrative secretaries to fulfill the administrative part of the patient admission.

The hospital physician examines the patient, although other hospital specialists could also be called (for instance, in this case, a surgery team). Considering the elements prescribed by the physician, nurses complete medical acts execution, and as the medical secretary, hospital physicians or 


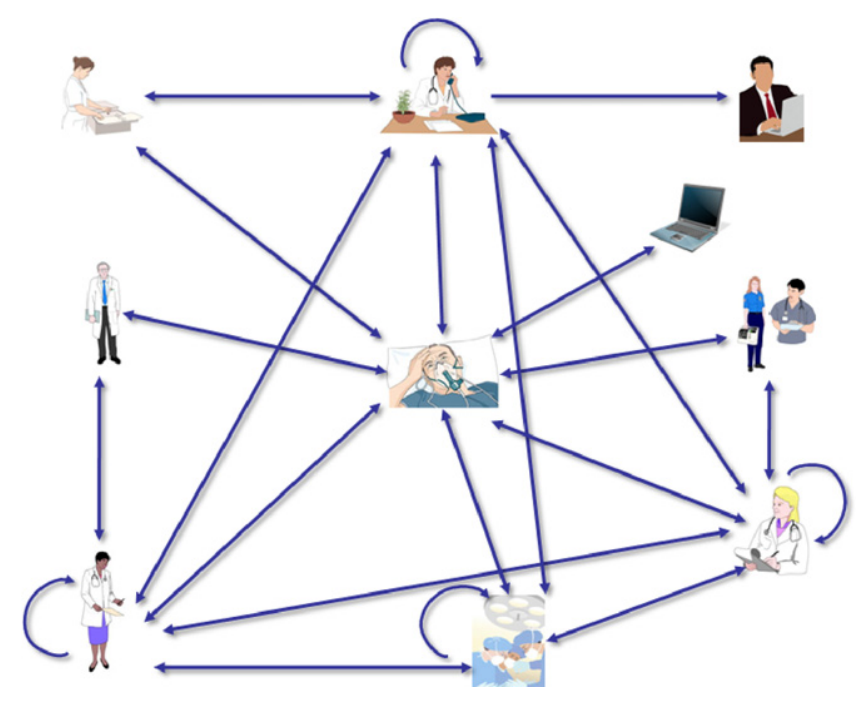

Fig. 2 - Identified information exchanges in a patient-centered scenario.

other specialists, may consult peers to accomplish their tasks. Reports of coded activities are transmitted periodically by the medical secretary to the hospital information department. The patient and/or family members are constantly informed of the care progress: medical examination, requested exams, pathology diagnosis, treatment decision, and how to handle it. Oral explanations and CPI sources are supplied regarding those items at the different stages of the care progress. Note that the patient or family members are expected to easily and permanently access CPI.

\section{Design considerations based on user requirements}

Medical services cover a wide range of activity patterns aimed at enabling proper healthcare provision. From this complex process, our field study concentrated on the main functions of what can be considered as a common patient-centered workflow of the visited services and technical units.

\subsection{Patient-centered care workflow}

It starts with the arrival of admitted patients (Fig. 3), who may come, once stabilized and diagnosed from emergency (Fig. 3 step (1)), or ambulatory consultation (step (2)). If the patient record does not exist, it is created. When the patient comes from another medical service (step (3)), the identification numbers of existing HIS and specific medical service(s) record(s) are communicated to the concerned medical service.

The patient and/or relatives are informed about the existence of the patient-centered record and how to use it during the care process (steps 1, 2, and 3). When the patient enters, the service or technical unit is informed about recent record updates (step 3). The medical secretary searches the service patient record, updates required fields, and adds new letters or reports (step 4). The physician studies the patient record, examines the patient (step 5), and decides if it is necessary to prescribe complementary exams done by a technical unit (step 6), or if existing results can lead to a therapy decision (step 7). At this point, in complex, rare, or difficult cases the physician can ask for a second opinion, search additional information in specialized databases, interrogating a decision support application if it is available [34-38], to confront the decisions. Otherwise, diagnosis and treatment are defined, discussed with the patient, and the corresponding patient record updated (step 8), before planning the corresponding patient care (step 9), as well as preparing and applying the prescribed medical procedures (step 10).

Nurses carry out prescribed physiological exams, administer medication, and collect data to update the patientcentered record, inputting relevant observations and comments (step 11). In a surgery context, nurses also prepare instruments and document operation room conditions for the surgery report (step 11). The anesthesiologist evaluates the patient before surgery, plans individual anesthesia procedures and post-operative support, notes periodically during surgery the patient physiological data that will be added to the surgery report (step 11). At the end of any medical procedure, the physician dictates a procedure report (step 12), which is coded to be sent to control entities (step 13) and to the referring physician (initially the family physician - step 14). If necessary the updated patient record is transferred to another clinical service (step 15). Thereafter, prescriptions are given and explained (step 16), and billing details are completed by the administrative secretary (step 17). When the patient leaves, historic data are stored (step 18) and a copy of the stay record is archived by the medical information department (step 19). Once a legal deadline is met, the concerned records are destroyed (step 20). Paramedics, pharmacists, and other medical personnel also participate at different steps of the described workflow. At each stage of this scheme, the patient and/or relatives are informed in detail about the care process and the available CPI. The proposed approach relies on this workflow to define the development framework, focusing on information exchanges related to patient-centered care.

\subsection{Roles of patient-centered workflow actors}

Information exchanges in the described patient-centered workflow take place according to well-defined actors' roles. These roles are described hereafter in a schematic manner, outlining the predominant patient-centered information processing related activities:

- Patient: uses frequently the patient-centered record before and after consultation, as well as before, during, and after hospitalization to improve personal understanding of the provided care, diagnosis, evolution of symptoms and treatment results, medication secondary effects, suggested lifestyle changes and legal advice, along with information exchanges among healthcare actors.

- Administrative secretary: collects administrative patient data concerning health insurance and billing. Sends pertinent information to medical services and technical units, following patient movements during hospitalization until it finishes. If necessary, gives administrative procedures status to the patient. 


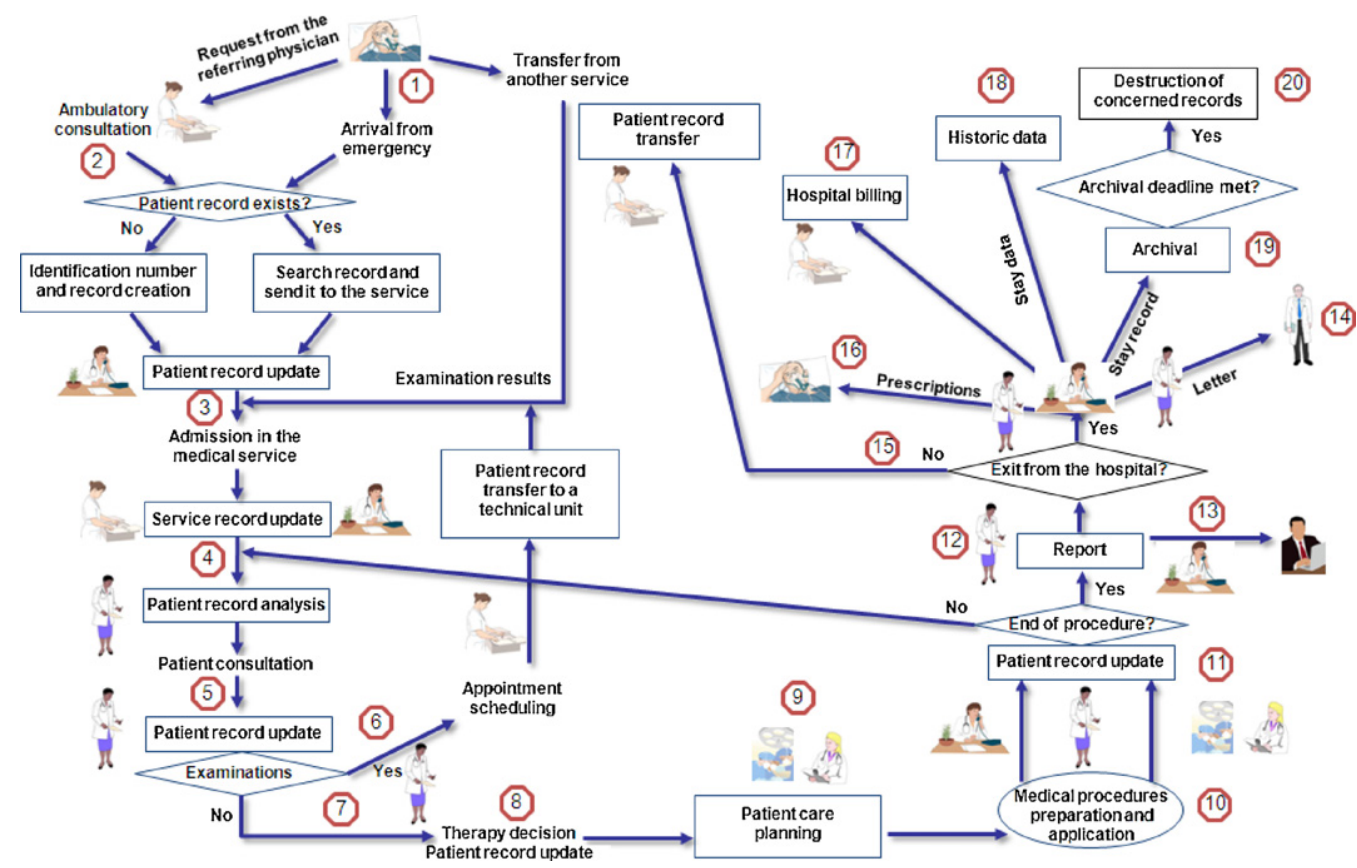

Fig. 3 - Main steps of the identified common patient-centered care workflow.

- Medical secretary: assigns a unique patient record identification number (PIN), and inputs patient medical data. During patient stay at the service, centralizes information for medical personnel about applied healthcare protocols, and all related CPI to be attached to the corresponding patient-centered record. When the patient leaves the service, sends the consultation and/or therapy reports to the HIS and the data storage service, making it available for other services, medical units, and the patient. Informs the patient and relatives about these actions and the nature of the available CPI.

- Hospital physician: access, visualizes, and modifies the patient-centered record. Examines the patient and provides all the necessary information about the diagnosed pathology, the prescribed therapy, other existing therapies and medication secondary effects, as well as particular lifestyle recommendations associated to the patient's clinical condition. Decides with the patient which healthcare protocol to apply and informs medical personnel. Writes consultation and/or therapy reports destined to the referring physician, keeping a copy in the service and patient records. Exchanges observations and opinions with other hospital physicians and the referring physician about the patient status. Points out all the necessary keywords to enable the search, classification and delivery of CPI for the patient. Answers patient questions and fosters common decision making.

- Nurse: accesses and visualizes patient records to apply the prescribed healthcare, updating the nursing section of the record, and informing the patient about given care. Communicates constantly with medical service personnel, physicians, and the patient.

- Referring physician: refers the patient to the medical service, providing requested parts of the patient record and any other useful information. If necessary, manages the prescribed treatment. Carries out medium and long-term follow-up of the medical service interventions and receives copies of consultation and/or therapy reports. Is authorized by the patient to access part or all the patient-centered record. Gives additional information to the patient and makes sure that the examination, treatments, and followup are properly understood.

- Paramedics, pharmacists, and other medical personnel: provide emergency medical care, and/or perform emergency pre-hospital life support interventions under physician supervision. Provide prescribed medication and devices required during hospitalization, among others. These personnel need critical information like allergies, treatments in progress, dietary restrictions (meals distribution), and pathologies, to improve provided care outcome. Explain unclear points of their actions to the patient.

- Internal and external control entities: exploit medical activity parameters, while supervising and regulating the way those values are generated. Conducted studies include analyses of patient-centered record adequacy and utilization.

\subsection{Requirements of patient-centered record users}

Except for paramedics, pharmacists, support personnel and control entities, all other described actors appear as core users of the patient-centered record. While the patient and the hospital physician proceed commonly as individuals, the members of medical services and technical units (including the concerned physicians) work as teams with common needs. For this reason, the most significant user requirements are listed and explained for patient, hospital physician, medical services, and technical units as related functional actors, even if some requirements may not be considered as strictly related to the patient-centered record. 
- Patient record creation: should take place only once at the HIS level for each patient, avoiding data redundancies.

- Patient record access: visualization of a summarized and comprehensible version of the patient medical information structured in a detailed chronological manner, by pathology, or selected periods of time. It should include consultation, diagnosis, therapy, surgery, laboratory, and paramedical reports, as well as acquired medical data.

- Patient record update: input of additional information on symptoms, reactions to medications, and personal treatment follow-up.

- Structured reporting: predefined report model useful for data aggregation, exchange, and analysis. Such report model is different depending on the concerned users (hospital physician, medical service, or technical unit).

- Access rights definition: decision made by the patient, about which parts of the record can be accessed by other workflow actors, or not.

- Information availability: the patient-centered record should be accessible $24 \mathrm{~h}$ a day and 7 days a week. Such service could be provided by a medical data storage service provider.

- External transmission: make available and communicate the EHR to the family physician or expert in another hospital, as well as the data storage service provider.

- Internal transmission: make available and communicate the EHR to other physicians or services that are also treating the patient.

- Confidential information: provided by the patient and unknown to relatives, or supplied by family members or medical personnel, without the patient being aware of it.

- Complementary information: for the patient, it consists in making use of selected information regarding the diagnosed pathology, the prescribed therapy and other existing therapies, secondary effects, particular lifestyle recommendations associated to the clinical condition, and the legal texts about patient rights. For the physician and medical services it consists in setting up the corresponding information, including the production of relevant documents, organizing the pieces of information available as CPI resources at the hospital level, pointing out information to be attached to a given record, and to be updated with respect to previously described patient record content. CPI is individually collected for each patient and contains basic parts (common for groups of hospitals and equivalent patient categories), combined with more specific pieces of information to be added depending on the treatment and/or pathology evolution, specific requests of the patient, and physician suggestions.

- Patient movements: follow-up of the patient consultations in other services and technical units of the same hospital.

- Appointments: management of patient appointments dynamic scheduling, taking into account patients' referral, along with experts and medical service constraints.

- System help: the patient needs a comprehensive, simple and clear help to access and update the patient record, as well as to access the CPI and appointments. The physician, medical service and technical unit members require support to properly make use of the patient record and its associated tools.

\begin{tabular}{|c|c|c|c|c|}
\hline & Patient & Physician & $\begin{array}{l}\text { Medical } \\
\text { Service }\end{array}$ & $\begin{array}{c}\text { Technical } \\
\text { Unit }\end{array}$ \\
\hline Patient record creation & \multicolumn{4}{|c|}{00} \\
\hline Patient record access & 0 & 0 & 0 & 0 \\
\hline Patient record update & 0 & 0 & 0 & 0 \\
\hline Structured reporting & & & & 0 \\
\hline Access rights definition & 0 & 0 & & \\
\hline Information availability & 0 & 0 & & \\
\hline External transmission & & 0 & 0 & \\
\hline Internal transmission & & 0 & 0 & 0 \\
\hline Confidential information & & 0 & 0 & 0 \\
\hline Complementary information & 0 & 0 & 0 & \\
\hline Patient movements & & & 0 & \\
\hline Appointments & 0 & & 0 & 0 \\
\hline System help & 0 & 0 & 0 & 0 \\
\hline
\end{tabular}

Fig. 4 - Correspondence between the four examined user types - patient, physician, medical service, technical unit and the previously described requirements.

Fig. 4 represents in a schematic manner the four main users' particular requirements, enabling to easily identify on each column the requirements subsets, cited by the respective examined actor.

This analysis does not pretend to be exhaustive, considering the complexity of defining a common workflow in the highly interdependent framework of a patient-centered record. The collected information provides nevertheless significant elements to formulate our approach.

Comparatively evaluating the described users' requirements allows identifying key functional features of the envisioned patient-centered record development framework. As it was previously identified in the theoretical patientcenteredness analysis (Section 2.1), the most important requirement is related to CPI, which is produced by physicians and medical services, to be accessed and understood by patients and/or their relatives. Associated data should be as complete and pertinent as possible, with respect to the particular situation of the concerned patient, in the sense of carefully selecting pieces of information in close relation with both the given patient record content and the temporal evolution analysis of the patient condition, treatment follow-up, control exams, etc.

Focusing on the practical notion of CPI, its agreement with the three selected components of the Mead and Bower model relies strongly on their translation into key functional objectives like:

- Provide the patient a reliable information source to search for details of interest (avoiding searching information from unreliable sources, e.g. the Internet).

- Guide the patient to prepare discussions with the physician: raise appropriate questions, being able to explain specific points to be detailed for a comprehension of the current situation. 


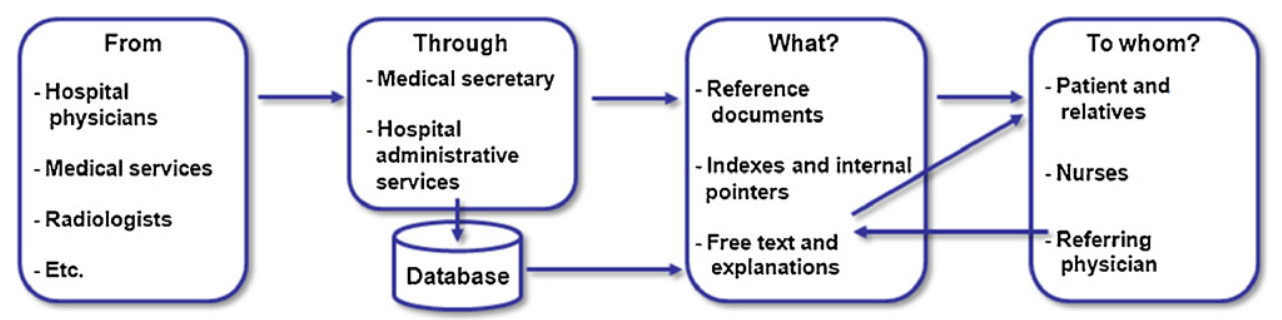

Fig. 5 - Actors and type of data involved in the production and utilization of CPI.

As a result, three other roles can be identified in relation with the question of CPI (Fig. 5): information producer, information organizer and supplier (person who transmits the information between the two other categories), and information receiver. The main actor likely to produce those information contents is the hospital physician. Writing such documentation is not evidently a common task in the daily medical practice of the hospital physician, but initially it could be assigned to volunteer practitioners, including specialists of a given domain (e.g. radiologist, staff from medical services and/or technical units, lawyers, etc.). However, the hospital physician keeps the essential responsibility of coordinating all actions related to producing documentation destined to the patient.

After reference documents are produced, CPI becomes part of medical information to be managed within the patientcentered record. The medical secretary or the administrative services gather specific pieces of information that correspond to each patient case according to the hospital physician indications, organize and attach the concerned documents and content to the record, and/or transmit that CPI directly to the final addressee. The patient must be told of this information availability.

Such a scenario raises the question of who else should be informed about the availability of CPI besides the patient or a family member, to facilitate the patient's understanding of its content. Among the actors listed above, only the nurse seems to be able to take that responsibility during the patient stay, but the referring physician can also have a significant role during the out clinic stage. At a functional level, oral explanations given directly to the patient during a discussion might be for instance strengthened by textual comments, emitted by nurses, hospital physician or referring physician, and joined to the general documentation as a way of personalizing CPI. Other well established information processing activity patterns, linked to budget execution, expending analysis, healthcare quality evaluation, and coding of medical activities, are also part of some identified actors' role, and could eventually affect patient information exchange if invested resources are diminished. They are not discussed here because except for medical activities coding, all of them take place outside medical services.

\subsection{Technical requirements for the management of complementary information}

Independently of the form given to CPI (e.g. documents, database entries, indexes, etc.), its content consists of structured and detailed text, diagrams and images, which imply a high workload for the actors who have the responsibility to write it. This charge will be very significant for the hospital physician, who according to the proposed model has a crucial role of coordinating the CPI providers. In addition to expert knowledge contributed by specialists, this conception task could also take advantage of existing medical knowledge databases. For instance, establishing automatic links between identified CPI keywords and concepts used in the indexation of global sources of evidence-based medicine, like the systematic reviews of the Cochrane library $[35,36]$, would facilitate ensuring that the concerned subject is further explored. Nevertheless, documents extracted from such information sources, not originally intended for patients, cannot be used directly as CPI, and a tradeoff remains to be found between completeness of the documentation and required effort to build and feed the information base. Furthermore, CPI management is also constrained by some technical considerations such as storage volume, information research efficiency, and servers' traffic, which highly influence data structuring. Depending on implementation resources and existing infrastructure, those system design decisions can be critical because just few storage, research and traffic models would be adapted to each particular case.

Consequently, taking account of these requirements in the proposition of a technical framework for managing CPI also depends on answers to the following questions:

- How basic documentation should be generated, which formats should be used, for which storage modalities, and according to which information research modalities?

- How to manage the different aspects of the CPI in order to find a compromise between information completeness and data volume?

The considered directions to answer above questions rely on the notion of patient categories. Although previous requirements concerning CPI are fully compatible with the three identified dimensions of the Mead and Bower model, a specific conceptualization associated to each dimension depends on multiple patient preferences that change according to the healthcare scenario. When asked about their specific requirements regarding patient-centered record content, all 12 interviewed volunteer patients had the idea of accessing information, for example, about their pathology, the therapeutic procedure risks, alternative therapies, secondary effects of medication, lifestyle changes, palliative care, or legal rights in case of medical error, among others. While the relative 


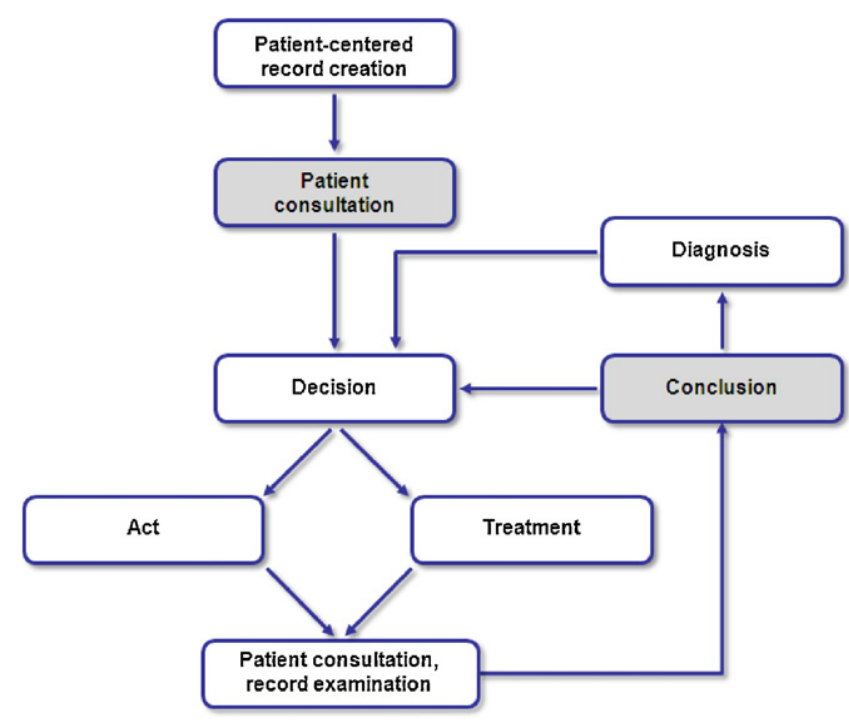

Fig. 6 - Attribution process to determine a patient category. At steps in gray (patient consultation, conclusion), the patient category has to be initialized and/or updated with the corresponding linked documentation.

importance of each item differs from one patient to another, the expected amount of information may also change depending on the pathology type and severity. Such variability was also identified in another context, with previously defined specific categorization of information preferences, related to information items and participation in medical decisionmaking [33]. Our work takes account of that variability by including concepts and attributes destined to express this categorization of patients' requirements in the resulting patient-centered record. Technically, the management of CPI is thus facilitated, with respect to above criteria, by abstractly associating necessary contents at the intersection of information classes (e.g. pathology description, medication, laboratory exams, prescribed treatment, etc.) and patient categories, defined with respect to the patient evolving situation.

However, the definition of specific patient categories is not considered in this work, because of the multiple links to contextual decisions taken in this sense by the hospital physician. Therefore, our patient-centered record model remains generic, having the possibility of being adapted to patient category types, depending on medical (type of pathology, severity, evolution stage, sex, age, degree of autonomy, etc.) or temporal elements (partial outcome of the therapeutic protocol), other patient indicators (computer literacy and education), or more subjective criteria in relation with the physician's experience (for instance the physician's perception of patient capabilities or post-treatment evolution potential, but also degree of interest in CPI and actual estimated capacity to make use of CPI).

In a typical scenario of patient-centered record creation (Fig. 6), no category is assigned to the patient until a first interview with the hospital physician has taken place. After initialization, the category assigned to a patient can be revised depending on the generated consultation reports, when medical acts and diagnosed pathologies are encoded. The assigned category then depends on both the current state of the patient-centered record and the history of coded medical acts, determining the detail level of CPI to be attached to the patient-centered record.

Otherwise, the task of structuring CPI to facilitate its research in large databases relies on keywords extraction and indexation mechanisms. Besides, the ability to search for pieces of information linked to a given subject will be useful for the patient, as well as for the medical secretary who assumes the role of attaching relevant documents to the patient-centered record depending on the medical condition. To support document selection, the most advanced form of information management that can be considered is the full automation of information attachment. Otherwise, intermediate information processing mechanisms can be imagined to guide medical secretaries, relying as well on matching between available documents on the CPI database and the patient record contents. Medical acts coding could play a key function to this end, given that codes represent directly an indexed version of the concerned medical information [34].

\section{Patient-centered record development framework}

In this section, we propose a development framework aimed at supporting the implementation of a patient-centered record. This technical analysis was focused on data structuring, examining how functional requirements expressed by interviewed users determine the record database design. The recommended data structures were conceptually defined, using a conventional entity-relationship (E-R) formalism, well adapted to model data destined to be stored into relational databases, widely used in the domain of medical information. This section presents the E-R schemas employed to structure data in the prototype that was implemented as a proof of concept.

\subsection{Main entities of the model}

The resulting data models form a multifaceted set of related entities, organized in 5 main components according to users' requirements and the identified data connections. Fig. 7 shows the core components of the patient-centered record: Person, Patient, Professional, Medical Personnel, and Hospitalization.

Person is the basic model to create, by inheritance, both Patient and Professional entities; a Patient undergoes a Hospitalization that is taken in charge by Medical Personnel. Hence, the core parts of the model representing an individuals' data, share an entity abstraction, destined to represent common aspects of the main actors identified in the workflow, but also to allow a coherent management of their numerous common requirements (Section 3). The Person entity corresponds to a separate data model (not pictured in Fig. 7), which stores identification, localization, and record access data, used by the Professional and Patient entities. The Professional entity generalizes the concept of health professionals, either Medical Personnel associated to medical Service or Unit entities, or Private Physician (not pictured) taking part in the concerned patient healthcare interventions, extending the question of 


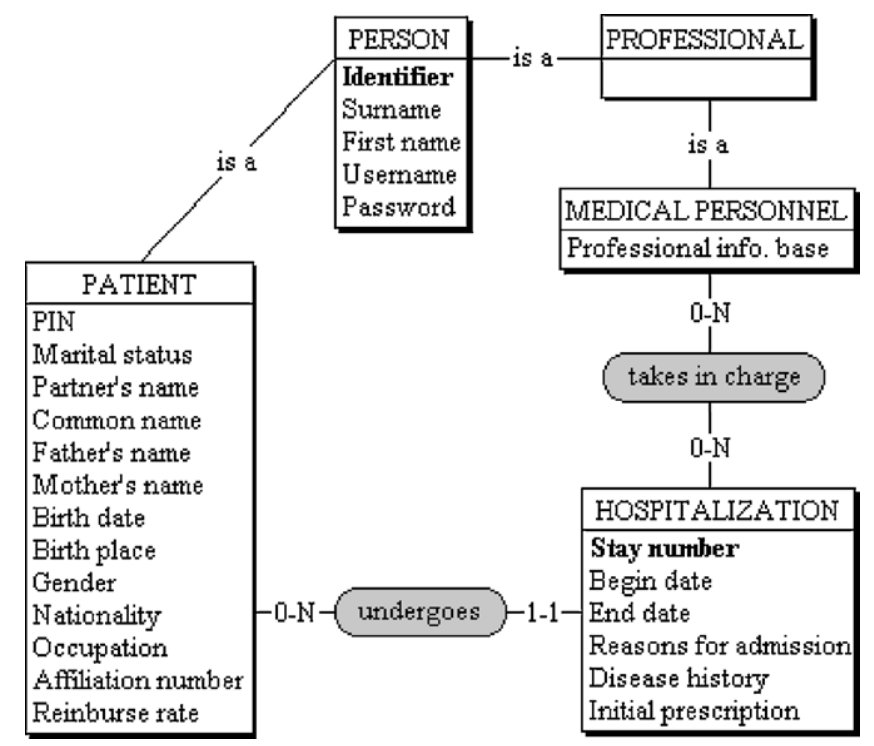

Fig. 7 - Example of the patient-centered record core components.

inheritance and the resulting data model cited above to a hierarchy of data types.

The need for coherent management of common requirements also relies on the notion of user groups, for instance adapted to structuring data related to a medical service or unit. Other applications of that notion are: a natural management of the list of professionals consulted by the patient outside the hospital, intended to be regularly updated to ensure their ability to refer to the record data and to update patient's follow up and treatment information, according to the "patient record access" and "patient record update" requirements; a support to the management of corresponding access rights at data level, and related actions on data, especially associated to the requirements of "access rights definition" and modification during "patient movements" and record "internal/external transmission" (e.g. granting access to a complete service, or to the role of medical personnel at once).

\subsection{Patient data model}

The Patient data model links conventional data components like specific medical data, personal history, pathology types, treatments, consultations, and acts (Fig. 8). A patient can go through ambulatory consultation that produces a record, be hospitalized, follow a treatment, go through an additional examination or a medical act described by the respective conditions, patient preparation, and known risks. These data are likely to be stored in distributed servers inside and outside the main reference hospital.

The requirement of "patient record access" expresses the need for both patients and health professional to access not only current record data, but also data that reflect the medical history of the patient in a chronological manner, implying the management of a temporal dimension in the relational data of the patient-centered record. History tables like Personal
History answer that requirement, with the additional benefits of providing an audit trail of record updates, the ability to extract useful aggregate numbers, and the possibility of recovering past record states or retrospective pathology search, if necessary [39].

\subsection{Medical Personnel data model}

Medical Personnel also have a complementary set of particular associated data entities (Fig. 9), in relation with patient hospitalization. During hospitalization, a medical intervention is carried out within a temporal frame. Each intervention corresponds to a set of acts, described initially using free text, to be later coded by means of tabular codes. Depending on intervention results and patient evolution, any decision and fact about a treatment (including unwanted effects) and medication, is documented in detail. CPI is labeled using keywords pointed out in the text part of the decision, referring to the information resources needed to fulfill the patient-centered system requirements.

During hospitalization and when it ends, conclusions reports are generated by the responsible medical personnel. These reports also contain relevant post-hospitalization keywords pointing to CPI documents considered necessary within the patient-centered scheme, and a given degree of confidence. According to the requirement of "structured reporting", pieces of information contained in these reports are arranged under the form of a conclusions summary, which can take the form of a conclusion type code and description list. No particular implementation is specified here for conclusion, decision and pathology type codes, to allow using different coding standards. Both decisions and conclusions generate letters sent to the referring physician (not pictured).

The temporal dimension linked to the requirement of "Patient record access" also induces the history of all requested and completed exams to be recorded in history tables (not pictured), as for patient's medical data, and requires constraints on temporal data to ensure, for instance, the coherence between related temporal frames and their compatibility with dates of hospitalization.

\subsection{Complementary information data model and technical specifications}

To comply with the patient-centered record users' requirements specifications defined in Section 3, CPI Elements (Fig. 10) have relations with medical data (not pictured) that translate the nature of CPI, summarized as follows:

- Selected information dynamically fitted to the patient evolving situation and degree of interest, to improve patient understanding of biomedical illness factors (associated to the Pathology Type entity): symptoms, pathology description and diagnosis procedure.

- Categorized information about therapies and surgical procedures, properly adapted to patient conditions, and patient questions, in order to facilitate decision making and 


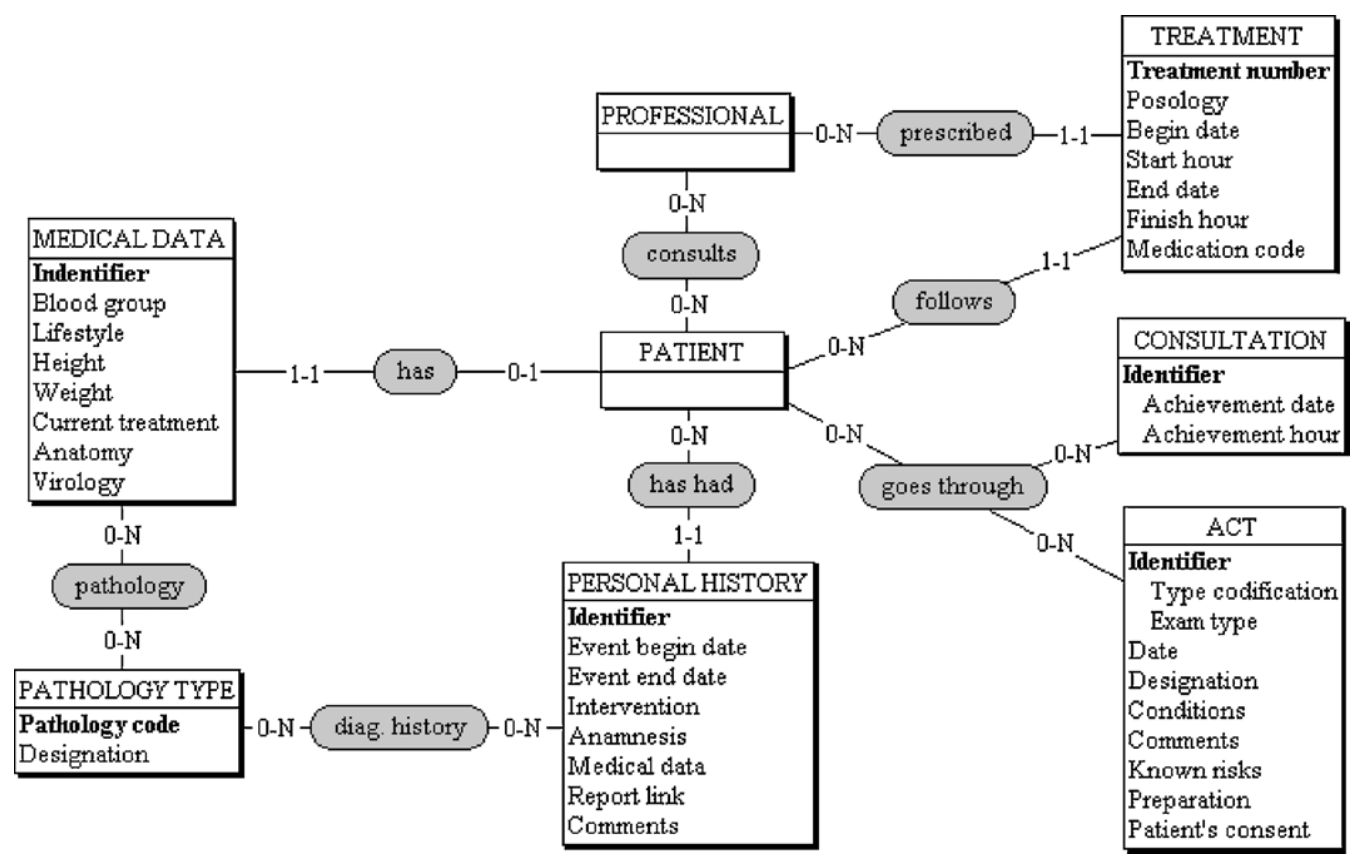

Fig. 8 - Conceptual Patient data model.

comprehension of therapeutic objectives (associated to the Act entity): treatment or surgical procedures descriptions, alternatives, hospitalization and discharge conditions.

- Treatment application description (associated to the Treatment entity): detailed explanation of the selected treatment procedure, precautions during treatment, possible side effects, and life style advice.

- Post-treatment information and requested patient participation (related in a distributed manner to the Pathology,
Act, and Treatment entities): once a decision has been taken, provide support for personal information acquisition, storage, and handling to enable personalized longitudinal follow-up, besides the physician and/or medical team follow-up, including recommendations about information delivery, post-treatment prevention, self-management for chronic illness, and palliative care conditions in case it would be necessary to treat pain, relief from suffering, or other distressing symptoms.

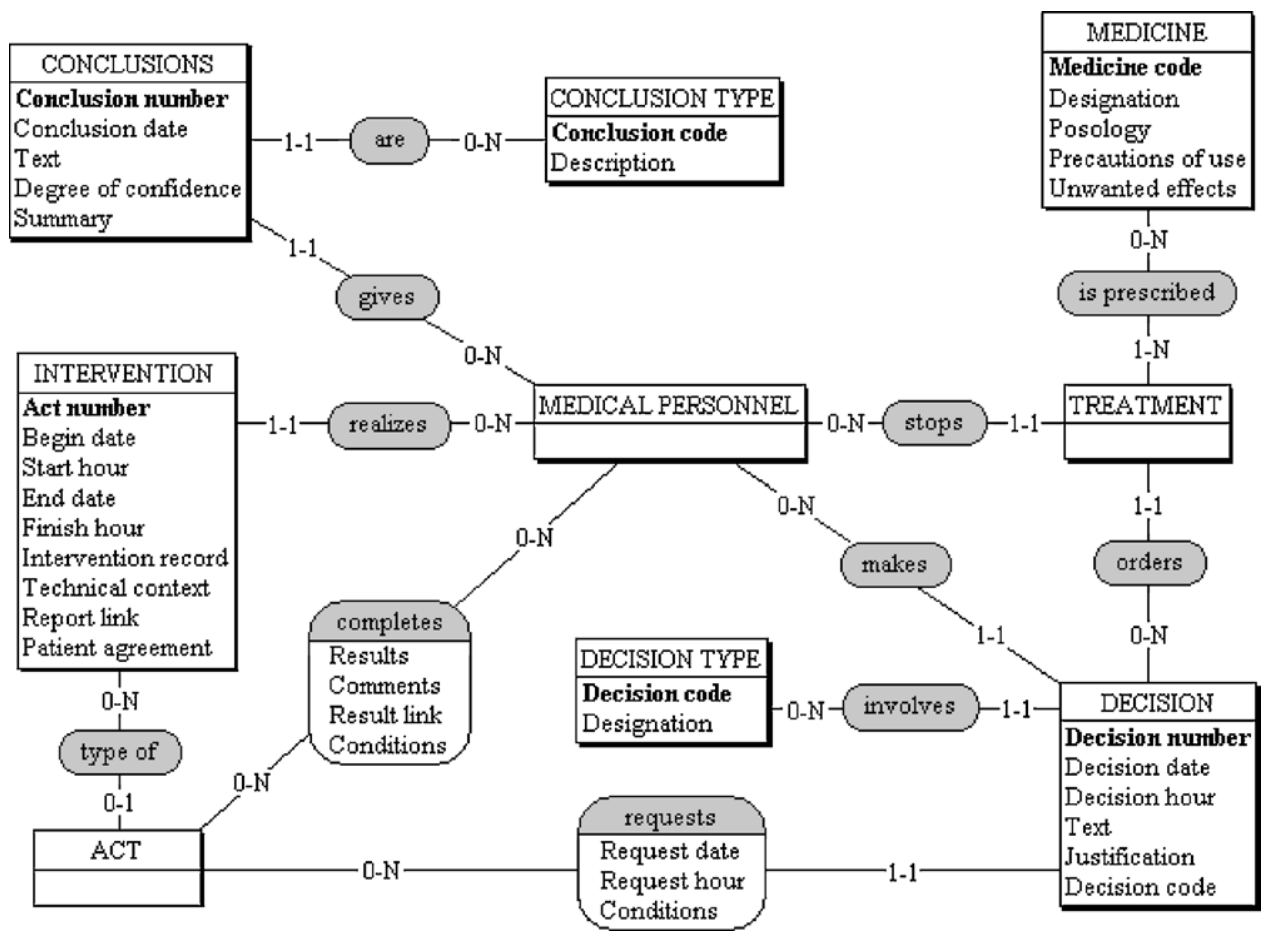

Fig. 9 - Conceptual Medical Personnel data model. 


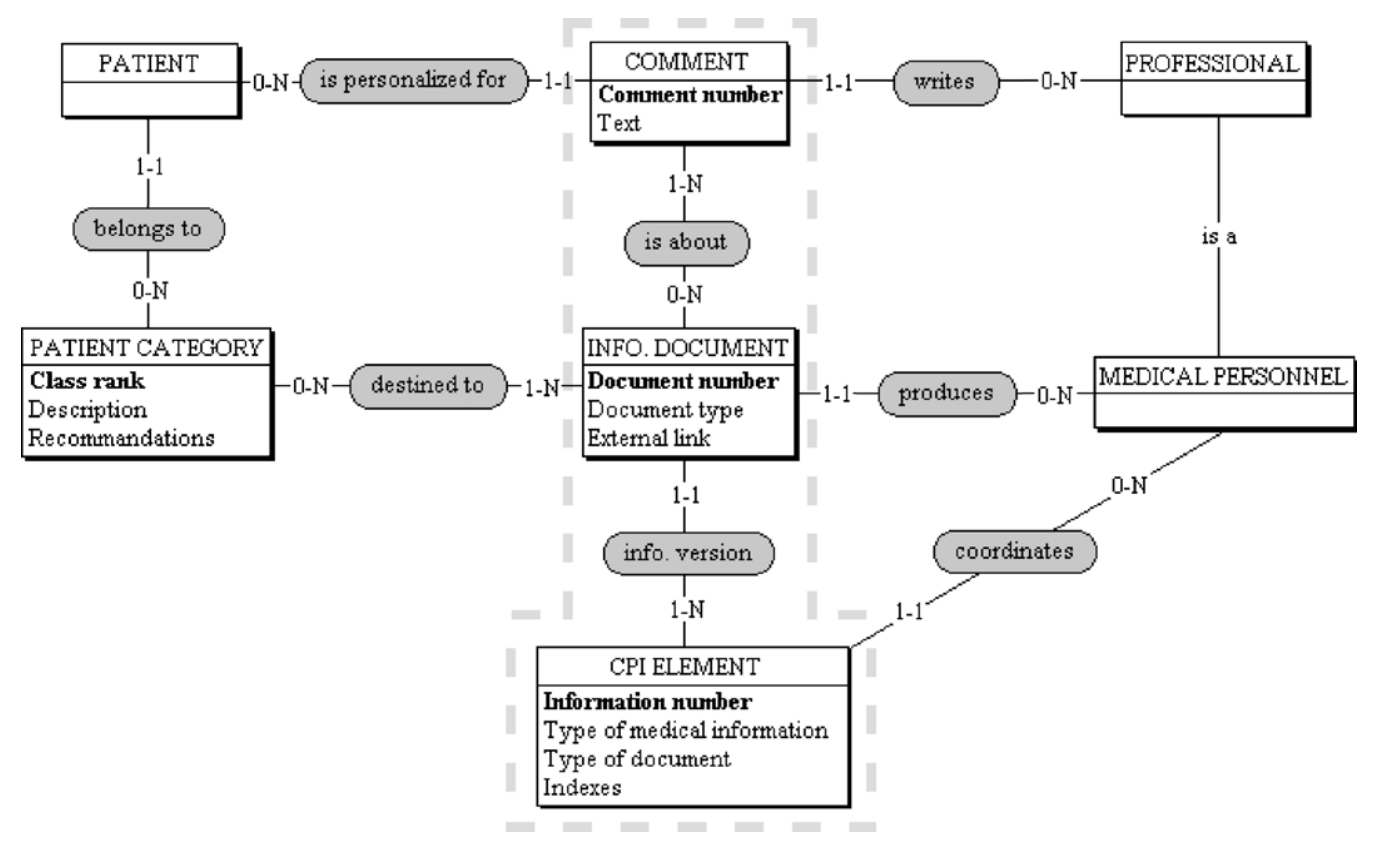

Fig. 10 - Entities related to the patient-centered record CPI model, centered on the E-R scheme proposed to manage information modularity (bounded in light gray).

Social and psychological factors did not emerge as part of the medical services and technical units patient-centered care awareness study, even though their inclusion could be important in some cases. Yet, those factors will be much better handled by a specialist in contact with the patient than by a system automatically retrieving information from a specific database.

Basic generic documentation concerning points cited above can then be enriched and personalized by the hospital physician or nurses in charge of the patient, or through follow-up by private physicians, via the addition of free text comments to information documents, concerning aspects linked to medical facts (e.g. relations between the patient life style and prescribed treatments). For traceability purposes, the identity of any comments author is kept.

When referring to complementary patient information, the term "information" designates informational contents like pieces of texts and specific ideas they convey, which have to be distinguished from documents, database entries, or digital files, used as media to store them. We assume that the nature of such information makes possible to exploit several support types and transmit it from information producers to information consumers. The proposed patient-centered record model is based on the assumption that CPI about a given topic is written in an electronic text file (e.g. in PDF format) that can be split into several parts or completed in an incremental way, depending on the target patient category. Such format will probably be more natural for information producers, and appropriate for patients to read. In addition, the requirement of CPI management makes necessary to join to these standard documents, free text as a support for both personalized information and explanation about CPI contents, and database entries that facilitate indexation and information research.
The data model on Fig. 10 also takes care of two technical requirements to examine: modularity in relation to the composed nature of the CPI and the ability to add dynamically new information entities. This last requirement corresponds to another inheritance situation (sub-types of CPI Elements in relation, as cited above, with pathology types, acts, etc.). The question of modularity refers to the reduction of coupling between parts of the information, to allow a separate evolution of, e.g. information documents and comments to the patient, both in terms of contents and form. We propose a simple data-structuring model to manage data modularity (Fig. 10), composed of the following entities: CPI Element, Information Document, and Comment. The separate evolution of each part is illustrated by the fact that versions and contents of Information Documents and Comments are adapted, respectively, to the patient category and specificity.

Whenever the record is modified (particularly when conclusions are recorded as shown in Fig. 6), attached CPI has to be updated, and information elements documented in relation with items detailed above, are retrieved in the database system by browsing relationships connected to the patient situation (record history, demographics data, and current clinical data) and therapeutic status (treatments, undergone medical interventions, decisions of the hospital physician and respective conclusions). Hence, those medical facts identified in the patient-centered record provide CPI, which is then declined into adapted documents, with respect to the patient category (or categories of lower ranks if this concept denotes a temporal evolution, justifying previous versions of information to be kept in the patient-centered record). A certain level of automation can also be considered to help the physician identifying key concepts and CPI indexation keywords, relying for instance on clinical tools used in the evidence-based medicine approach to emit alerts and reminders from input 


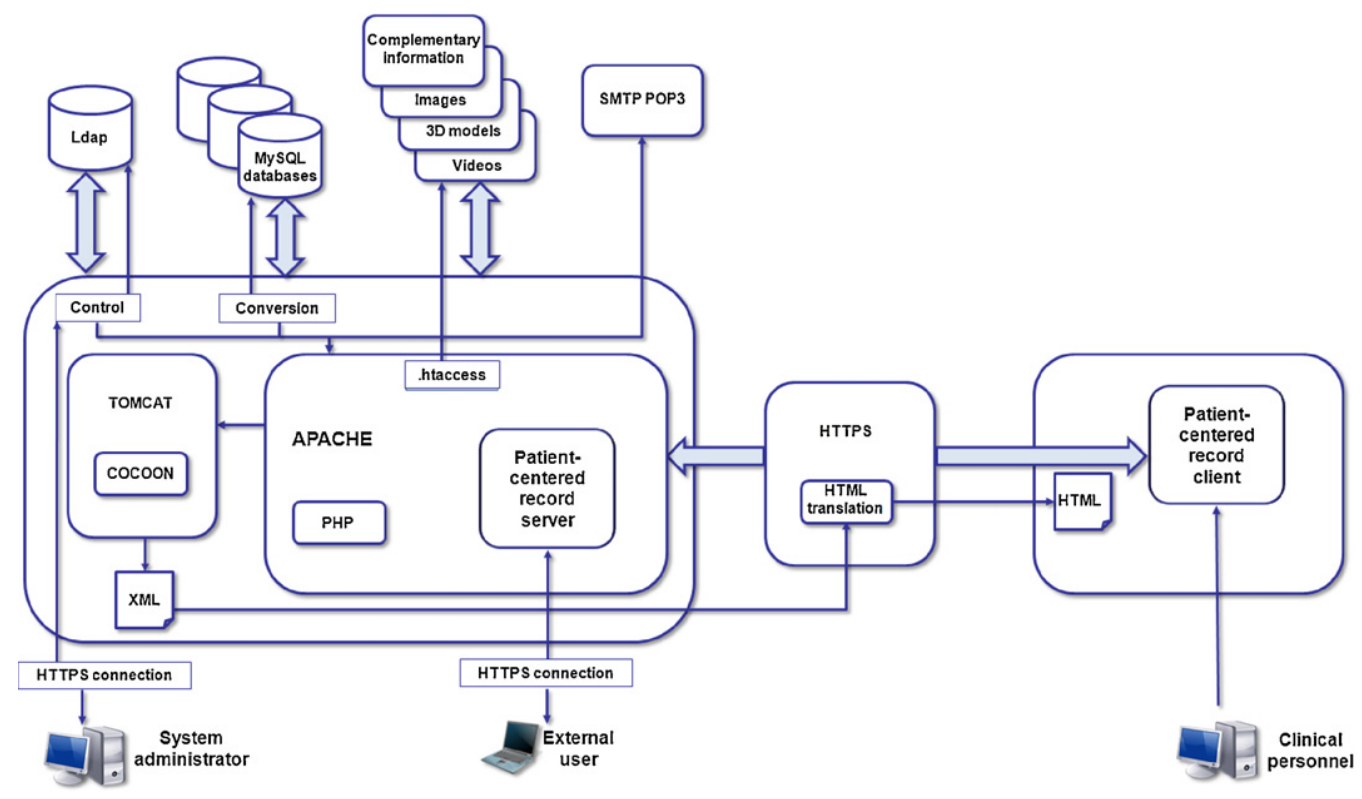

Fig. 11 - Patient-centered record client-server system architecture.

medical records $[37,38]$, and in relation with medical coding systems [40].

\subsection{System architecture}

Diverse architecture models like multitier, web-services, and client-server could be utilized to develop the proposed patient-centered record. A client-server architecture, integrating data management and application processing, was chosen according to an open source software approach. It did not consider however the issue of using different patient identifier approaches, namely the lack of PIN uniqueness. In the particular case of French hospitals, on which our study is based, the issue of patient identification has been partially solved through multiple separate local and regional initiatives [41]. We assume nonetheless that there is a unique PIN, used by all medical services and technical units of a given hospital, capable of assuring patient anonymity. User attributes were handled by a profiles database. A lightweight directory access protocol (LDAP) was defined for this purpose, considering its effectiveness to embody hierarchical entries representing individuals, groups, and systems, as well as to prevent unauthorized access to the system. Patient-centered records were structured applying the previously described data model.

\subsubsection{Client and server}

On the client side it is only necessary to run a web navigator on a personal computer connected to a network, without allowing any local storage of the consulted information. Content was encapsulated for presentation, using dynamic PHP pages and Java applets, which can be handled by any navigator, permitting to include supplementary security restrictions and controls.

Several components were required on the server side: web server, content handling and presentation scripts, databases, and database management system (DBMS). An Apache web server, a PHP script processor to create dynamic web pages, a Tomcat-Cocoon based XML-XSLT content manager, and a MySQL relational DBMS, were the implemented components. The database was designed to handle increasing data volume and multiple kinds of data formats, including references to multimedia objects (documented images and images sequences).

\subsubsection{Modules connection}

The patient-centered record architecture was designed to be open, modular, flexible enough to facilitate changes, and based on various standards. Its main objective is to easily permit the addition of other databases and functionalities, without having to redefine significant parts of the system. Our implementation proceeded in this direction making use of widely accepted, developed, and utilized informatics standards. Fig. 11 illustrates the main system architecture components on the server and client sides. The common trait of most user requirements is the patient-centered record utilization, which may take different forms depending on how it is exploited. Selected parts of the patient-centered record or all available information are displayed depending on user rights, when the respective application user interface is activated. System administration functions were also an essential part of the patient-centered design, even though they were not been explicitly addressed in the previous sections.

\section{Discussion}

This work explores a computerized patient-centered record development framework for scenarios on which it is necessary to manipulate information units formed by specific CPI and patient data, which can be independently stored, transmitted, accessed, and visualized. According to this principle, the proposed patient-centered record model looks for the manner 
to build an articulated system, using information modules on data repositories, enabling transversal information streams exchanges between medical services and technical units, avoiding redundant information. It simplifies the integration of other data types and applications to the defined architecture, in the case of further system development.

From a technical point of view, future implementations of a patient-centered medical record based on the proposed framework will have to structure data and related procedures according to users' requirements and to the technical analysis that was made of these user needs (Section 4). Relational schemas proposed as practical solutions to these functional requirements are domain-specific, but some key concepts identified from the workflow and its analysis can be abstracted to commonly occurring data-structuring problems, leading to define and apply more general database design patterns. Database design has been experimented directly transposing concepts of software design patterns, known to facilitate capitalizing on accumulated knowledge and experience to ensure extensibility and maintainability features [42,43], aiming at defining data models at the conceptual, logical, and physical levels [44]. Original relational database patterns have also been proposed, mostly formalized by E-R schemas, for the formal expression of conception rules [45]. In addition to advanced database patterns aiming at implementing properly inheritance in relational data, the following key data-structuring concepts identified from users' requirements can be associated to structuring patterns: extension of the notion of inheritance to a hierarchy of data types ("Hierarchy" pattern), associated to a generic management of the notion of user groups ("Composite" pattern); this last pattern is also adapted to structuring data associated with predefined report models, possibly different for each kind of medical actor, for the purpose of generic processing. In that case, the required separation between data interface and implementation can also rely on advanced solutions to manage in a modular manner CPI entities ("Materialization" pattern, inspired from the principle of the "Bridge" software pattern). Also, adding a specialist from a health profession not yet registered in the record, or a new kind of CPI element, can justify the creation of additional relational data fields when characterized by specific attributes ("Pivoting" pattern). Finally, the management of temporal frames for entities such as Intervention and Act requires the design of corresponding data storage and management strategies [39].

Besides those technical questions, we assumed that patient-centered care can seamlessly make use of a patientcentered record, although several questions need to be further examined in detail. Prospective medical personnel views were taken as reference to guide system development, given that under the proposed framework they are expected to identify and collect all the needed CPI to be stored and displayed by the patient-centered record application. As a consequence, a patient-centered record demands more physician involvement to prepare, select, and define CPI attribution according to diagnosis, therapy, and clinical condition. Is it reasonable to add that task to physicians' responsibilities? Moreover, other professionals participation will also be necessary, for instance to provide patients' legal rights, lifestyle changes, or psychological information. On the other hand, the provision of selected CPI implies significant investment from medical institutions, which would be expected to support CPI creation and utilization, instead of letting the patient struggle alone to eventually find it. Will hospitals be in a position to efficiently develop and handle those information services? An increasing number of hospitals have been proposing simplified information about medical exams descriptions on their web sites. The next step toward an elementary patient-centered record approach could be to conveniently link that information to specific patient accounts, according to diagnoses and therapies.

Since patient education level is not uniform, what information does the patient need to build-up proper understanding? Answers to this question are fundamental to pre-select the type of CPI that should be provided according to the patient condition, as well as to determine if targeted educational efforts can reduce patient lack of understanding. Should the patient be authorized to access all the patientcentered record information? Some physicians suggested that it must rather be a summary, eventually containing several images. Nevertheless, a summary represents redundant work, unless it would be automatically extracted from the patient record. This fact raises additional questions about which kind of language would be employed, how to structure the original documents, and how detailed should summaries be. The question about access rights is even more complex given that under certain circumstances, and despite patient decisions, information should be disclosed if it can ameliorate an intervention result, for instance in emergencies.

The question regarding the necessity of including multimedia data in the patient-centered record is debatable. Legal and medical reasons require archiving during pre-defined times, diagnostic data like medical images and signals, as well as laboratory results and medical prescriptions. However, once the diagnosis has been completed, the physician rarely examines again the complete multimedia data set, except for the consultation reports and the patient history. Also, it is not clear how important will multimedia content be for the patient. Alternatively, patient-centered care leads to the interaction of several actors or detailed patient follow-up, making medical activity partially dependant on the availability of multimedia data, shared in a distributed infrastructure. The proposed platform copes with this functionality.

Another essential question thus remains concerning the issue of generating complementary documentation, under the constraint of producing reusable documents and information to reduce the workload associated with this task. Whether such information is structured as fields of a database or simply compiled into files, as supposed in the defined architecture, reusing it should be easier than exchanging patient information, since CPI is not submitted to confidentiality constraints or equivalent restrictions. However, access to raw information should be restricted to professional use, given that in the context of the patient-centered record, reliable CPI is of key importance. A CPI document repository should be built therefore with standard website architecture. The only condition to be fulfilled for an efficient sharing of content will be the existence of common previously defined patient categories, having a semantic meaning shared by different specialists, 
whose sensitivities with respect to the patient classification problem may differ.

It is also important to consider the ability to browse CPI associated to a given record. Whether this information is stored under a relational form or is presented as a set of referenced documents, indexing can be used to link keywords extracted from the patient record with relevant information. Therefore, some uses of the indexing mechanism could be:

\section{- Automatically associate (fully/partially) referenced docu- ments to the record. \\ - Search particular information within the complementary documentation. \\ - Create internal links between parts of documentation asso- ciated to a given record, allowing the patient to browse information, and increasing the flexibility of relations between referenced documents and patient categories. \\ - Dynamic creation of alternative reading paths within the added CPI, depending for instance on the degree of detail, or particular medical aspect.}

We also note the importance of using standardized keywords and concepts for information indexation, possibly common with existing medical knowledge and evidencebased medicine databases (e.g. Cochrane library, EBMeDS), potentially simplifying the conception of shared CPI repositories cited above, and possibly facilitating the use of semi-automatic CPI generation tools.

There are other technical unit systems, like pharmacovigilance (prevention of short and long term adverse side effects of medications), vigilance of human parts (organs, tissues, and cells) utilization as medical treatments, and vigilance of nosocomial infections (developed after patient admission at the hospital), which have not been considered in this study, but that could also be part of patient-centered record design.

\section{Lessons learned}

Despite the existence of multiple patient records types [46], these are essentially physician-centered or medical servicecentered, and as a consequence do not enable patients to completely and easily understand their medical conditions and healthcare process. If such understanding could be facilitated by means of a patient-centered record, it implies a mandatory transfer of the patient-centeredness notion from medical practice to medical practice support systems, by developing adapted information technology applications, tailored to medical workflow. On the other hand, previous patient-centered record studies documented in the literature, basically represented the dimension of patients' access to part of their personally generated medical data, according to individual choices. In this context, partial views of the original physician-centered or medical service-centered records are extracted and visualized, without making use of associated CPI.

Our work identified most necessary elements to define the requirements of a patient-centered record through interviews, field data collection and analysis of patient-centered awareness, leading to the conception of a development framework, to collect and select adapted complementary patient-centered information. In this sense, the requirements study focused on patient-centeredness awareness by exploring its interrelated dimensions (information exchanges, patient-centered care workflow, roles of main actors, user requirements, type of patient-centered record and technical requirements for the management of CPI), often neglected because of the previously mentioned predominance of physician-centeredness.

One of the main insights from this study is the indication that a patient-centered record mainly contains CPI, which must be dynamically adapted to the patient evolving situation, depending on abstract categories intended to link related documentation. Multiple questions related to the way of producing and handling appropriate CPI result from this analysis. Given that all patients are expected to make use of a personalized patient-centered record, compatible with their past and current medical status, along with what could be considered an arbitrarily assigned capability to produce (for medical personnel) and to handle (for patients) the provided information, the automated treatment of the whole process is likely to be complex.

As a consequence, improvement of how the patient understands and follows his/her own healthcare process requires additional specialized medical and non-medical personnel contributions, further system developments, and more detailed user requirements analysis, beyond the basic scheme presented in this paper. Moreover, patients do not know yet what to expect from a patient-centered record, other than be able to consult part of their medical information, decide who may have access to it, and examine their respective CPI.

\section{Future work}

Patient-centeredness is a well-known medical practice concept that requires specific user requirements and workflow analysis to be integrated in medical information systems. The proposed patient-centered record is based on hospital personnel/patient prospective interactions likely to take place during hospitalization or consultation, conceptualizing a set of coherent abstractions that share embedded patient oriented information, to complete the patient-centered care process.

Further work in this direction needs first to examine the efficacy of the developed data models, particularly the entities related to CPI, in order to find out up to what point data obtained from historic, consultation and treatment followup records, allow identifying the correct reference phrases to generate the associated CPI. Otherwise, versions of the implemented architecture using existing components and definitions from medical informatics standards should be examined in order to address the proposed framework adequacy in terms of constrained functionality. The next step will be to investigate automatic procedures to collect CPI information according to key words identified in EHR, and how those procedures cope with infrastructure restrictions.

It will also be important to measure the necessary effort asked to physicians and other professionals to generate the reference and more specialized CPI, which undoubtedly results in a workload increase for the concerned personnel. 
Furthermore, availability of such CPI implies that patient access to selected information must be dynamically configured by the system, depending on the correlation between specific patient requests and keywords marked by physicians' on the reports or automatically identified. Finally, regardless of the data increase compared to conventional patient record models, the patient-centered record could improve not only how the patient understands the healthcare process, but also enable physicians to stimulate patients in the process of becoming more active actors of their own health. The endeavor of measuring this impact will conduct probably to reformulate parts of the initial patient-centered record principles.

\section{Conflict of interest statement}

Authors declare not having any personal, financial, or additional source of relationships with other people or organizations that could inappropriately influence their work.

\section{REF E R E N C E S}

[1] N. Mead, P. Bower, Patient centredness: a conceptual framework and review of empirical literature, Social Science and Medicine 51 (2000) 1087-1100.

[2] A.A. Atienza, B.W. Hesse, D.H. Gustafson, R.T. Croyle, E-health research and patient-centered care. Examining theory, methods, and application, American Journal of Preventive Medicine 38 (1) (2010) 85-88.

[3] D.P. Lorence, R. Churchill, Clinical knowledge management using computerized patient record systems: is the current infrastructure adequate? IEEE Transactions on Information Technology in Biomedicine 9 (2) (2005) 283-288.

[4] J.F. Fries, Alternatives in medical record formats, Medical Care 12 (10) (1974) 871-881.

[5] C.J. McDonald, G.O. Barnett, Medical-record systems in medical informatics - computer applications, in: E.H. Shortliffe, L.E. Perreault (Eds.), Health Care, Addison-Wesley, Reading, MA, 1990, pp. 181-218.

[6] A.L. Rector, W.A. Nolan, S. Kay, Foundations for an electronic medical record, Methods of Information in Medicine 30 (1991) 179-186.

[7] H. Tange, How to approach the structuring of the medical record? Towards a model for flexible access to free text medical data, International Journal of Bio-Medical Computing 42 (1-2) (1996) 27-34.

[8] R.E. Rakel, The problem-oriented medical record, in: R.E. Rakel (Ed.), Essentials of Family Practice, W.B. Saunders, Philadelphia, PA, 1998, pp. 91-107.

[9] E. Berner, D. Detmer, D. Simborg, Will the wave finally break? A brief view of the adoption of electronic medical records in the United States, Journal of the American Medical Informatics Association 12 (1) (2005) 3-7.

[10] M. Eichelberg, T. Aden, J. Riesmeier, A. Dogac, G.B. Laleci, A survey and analysis of electronic healthcare record standards, ACM Computing Surveys 37 (4) (2005) 277-315.

[11] D. Wollersheim, A. Sari, W. Rahayu, Archetype-based electronic health records: a literature review and evaluation of their applicability to health data interoperability and access, Health Information Management Journal 38 (2) (2009) $7-17$.
[12] W. Goossen, A. Goossen-Baremans, M. van der Zel, Detailed clinical models: a review, Healthcare Informatics Research 16 (4) (2010) 201-214.

[13] D. Kalra, Electronic health record standards, in: R. Haux, C. Kulikowski (Eds.), IMIA Yearbook of Medical Informatics, International Medical Informatics Association, 2006, pp. 136-144.

[14] S. Garde, E. Hovenga, J. Buck, P. Knaup, Expressing clinical data sets with openEHR archetypes: a solid basis for ubiquitous computing, International Journal of Medical Informatics 76 (3) (2007) S334-S341.

[15] C. Martínez-Costa, M. Menárguez-Tortosa, J.T. Fernández-Breis, An approach for the semantic interoperability of ISO EN 13606 and OpenEHR archetypes, Journal of Biomedical Informatics 43 (5) (2010) 736-746.

[16] R.H. Dolin, L. Alschuler, S. Boyer, C. Beebe, F.M. Behlen, P.V. Biron, A.S. Shvo, HL7 clinical document architecture, release 2, Journal of the American Medical Informatics Association 13 (2006) 30-39.

[17] ASTM E 1384: Standard Practice for Content and Structure of the Electronic Health Record, 2007.

[18] V.J.M. Watzlaf, X. Zeng, C. Jarymowycz, P.A. Firouzan, Standards for the content of the electronic health record, Perspectives in Health Information Management 1 (1) (2004) 21, http://www.ncbi.nlm.nih.gov/pmc/articles/PMC2047330/.

[19] P.C. Tang, J.S. Ash, D.W. Bates, J.M. Overhage, D.Z. Sands, Personal health records: definitions, benefits, and strategies for overcoming barriers to adoption, Journal of the American Medical Informatics Association 13 (2) (2006) 121-126.

[20] A. Stolyar, W.B. Lober, D.R. Drozd, J. Sibley, Feasibility of data exchange with a patient-centered health record, Proceedings AMIA Symposium (2005) 1123.

[21] W.J. Donnelly, Patient-centered medical care requires a patient-centered medical record, Academic Medicine 80 (1) (2005) 33-38.

[22] K.A. Kuhn, D.A. Giuse, From hospital information systems to health information systems: problems - challenges perspectives, Methods of Information in Medicine 40 (4) (2001) 275-287.

[23] K. Davis, S.C. Schoenbaum, A.-M. Audet, A 2020 vision of patient-centered primary care, Journal of General Internal Medicine 20 (10) (2005) 953-957.

[24] D. Kjeldmand, I. Holmström, U. Rosenqvist, How patient-centred am I? A new method to measure physicians' patient-centredness, Patient Education and Counseling 62 (2006) 31-37.

[25] R. Henbest, M. Stewart, Patient-centredness in the consultation 1: a method for measurement, Family Practice 6 (1989) 249-254.

[26] J. Brown, M. Stewart, S. Tessier, Assessing Communication Between Patients and Doctors: A Manual for Scoring Patient-centred Communication, Centre for Studies in Family Medicine \& Thames Valley Family Research Unit, Working Paper Series, London, Ontario, 1995, pp. 95-102.

[27] J. Brown, M. Stewart, E. McCracken, I. McWhinney, J. Levenstein, The patient-centred clinical method 2: definition and application, Family Practice 3 (1996) 75-79.

[28] M. Berg, Patients and professionals in the information society: what might keep us awake in 2013, International Journal of Medical Informatics 66 (2002) 31-37.

[29] A.S. Rothschild, L. Dietrich, M.J. Ball, H. Wurtz, H. Farish-Hunt, N. Cortes-Comerer, Leveraging systems thinking to design patient-centered clinical documentation systems, International Journal of Medical Informatics 74 (2005) 395-398.

[30] J.J. Cimino, V.L. Patel, A.W. Kushniruk, The patient clinical information system (PatCIS): technical solutions for and experience with giving patients' access to their electronic 
medical records, International Journal of Medical Informatics 68 (2002) 113-127.

[31] W.S. Tuil, A.J. ten Hoopen, D.D.M. Braat, P.F. de Vries Robbé, J.A.M. Kremer, Patient-centered care: using online personal medical records in IVF practice, Human Reproduction 21 (11) (2006) 2955-2959.

[32] M. Wang, C. Lau, F.A. Matsen, Y. Kim, Personal health information management system and its application in referral management, IEEE Transactions on Information Technology in Biomedicine 8 (3) (2004) 287-297.

[33] K. Pardon, R. Deschepper, R.V. Stichele, J. Bernheim, F. Mortier, L. Deliens, Preferences of advanced lung cancer patients for patient-centered information and decision-making: a prospective multicentre study in 13 hospitals in Belgium, Patient Education and Counseling 77 (2009) 421-429.

[34] L. Lecornu, G. Thillay, C. Le Guillou, P.J. Garreau, P. Saliou, H. Jantzem, J. Puentes, J.M. Cauvin, REFEROCOD: a probabilistic method to medical coding support, in: Proc. 31st International IEEE EMBS Conference, Minneapolis, USA, 2009, pp. 3421-3424.

[35] A. Avenell, H.H.G. Handoll, A.M. Grant, Lessons for search strategies from a systematic review, in The Cochrane Library of nutritional supplementation trials in patients after hip fracture, The American Journal of Clinical Nutrition 73 (3) (2001) 505-510.

[36] J.P.T Higgins, S. Green (Eds.), Cochrane Handbook for Systematic Reviews of Interventions, Wiley-Blackwell, 2008, http://dx.doi.org/10.1002/9780470712184.fmatter.

[37] R.B. Haynes, H.P. McDonald, A.X. Garg, Helping patients follow prescribed treatment: clinical applications, Journal of the American Medical Association 288 (22) (2002) 2868-2879.
[38] J. Meddings, M.A.M. Rogers, M. Macy, S. Saint, Systematic review and meta-analysis: reminder systems to reduce catheter-associated urinary tract infections and urinary catheter use in hospitalized patients, Clinical Infectious Diseases 51 (5) (2010) 550-560.

[39] C.J. Date, H. Darwen, N.A. Lorentzos, Temporal Data and the Relational Model, Morgan Kaufmann Publishers, 2003.

[40] L. Lecornu, C. Le Guillou, F. Le Saux, M. Hubert, J. Puentes, J.M. Cauvin, ANTEROCOD: actuarial survival curves applied to medical coding support for chronic diseases, in: Proc. 32nd Annual International Conference IEEE Engineering in Medicine and Biology Society, Buenos Aires, Argentina, 2010, pp. 1158-1161.

[41] A. Perrin, C. Morin, Patient identification vigilance in public healthcare organizations: inventory in French hospitals and proposals, Annales de Biologie Clinique 67 (2) (2009) 177-189.

[42] E. Gamma, R. Helm, R. Johnson, J. Vlissides, Design Patterns: Elements of Reusable Object-oriented Software, Addisson Wesley, 1995.

[43] C. Larman, Applying UML and Patterns: An Introduction to Object-oriented Analysis and Design and the Unified Process, 2nd ed., Prentice Hall, 2001.

[44] C.S. Ku, T.J. Marlowe, T. Budanskaya, P.K. Kang, Software engineering design patterns for relational databases, in: Proc. International Conference on Software Engineering Research and Practice, Las Vegas, USA, 2007, pp. 340-344.

[45] N. Vitacolonna, Conceptual design patterns for relational databases, in: Proc. 17th International Conference on Information and Software Technologies, Kaunas, Lithuania, 2011, pp. 239-246.

[46] H.-C. Wen, Y.-S. Ho, W.-S. Jian, H.-C. Li, Y.-H. Hsu, Scientific production of electronic health record research, 1991-2005, Computer Methods and Programs in Biomedicine 86 (2007) 191-196. 